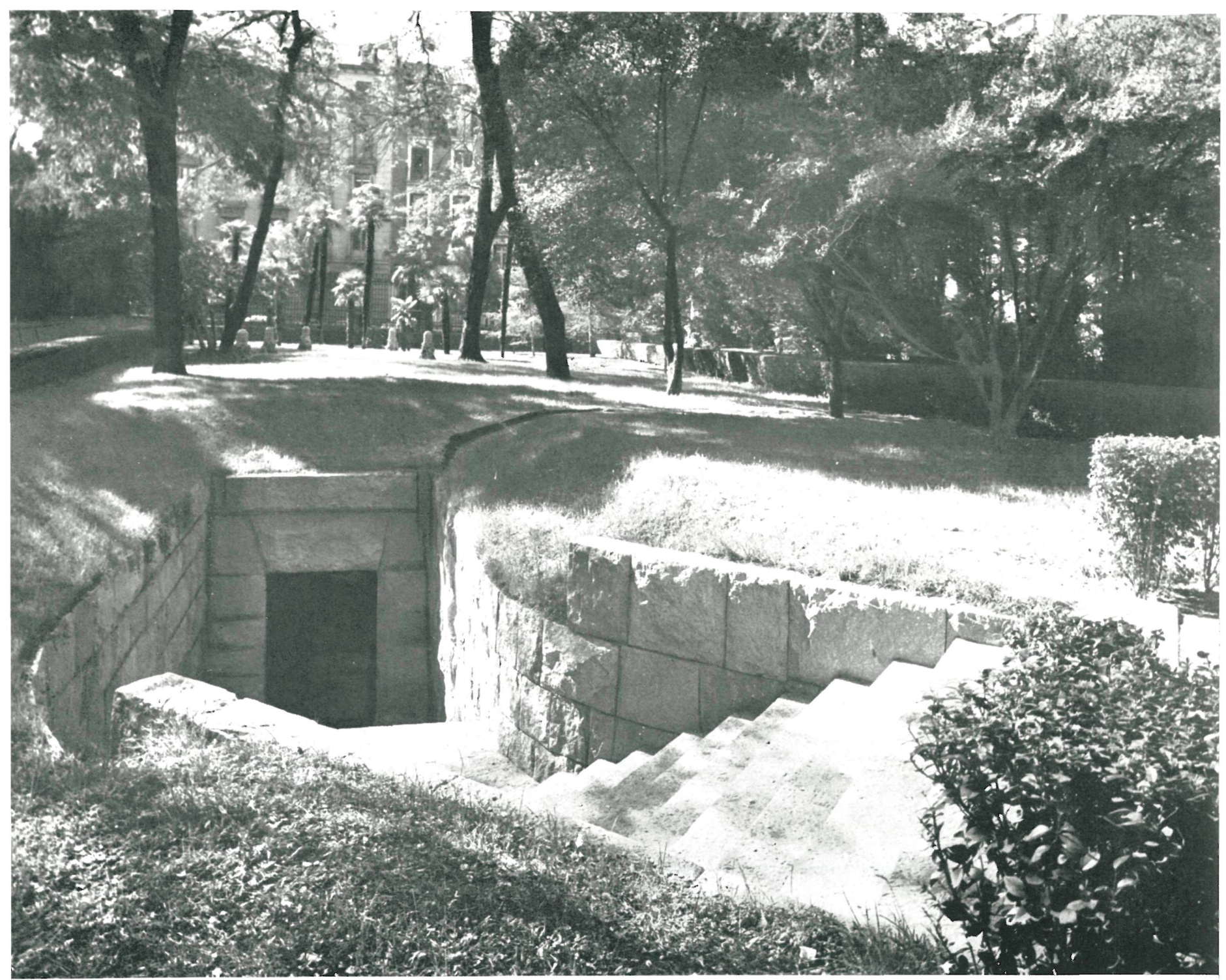

189.2

\title{
instalación en Madrid de una reproduckión de las pinturas prehistóricas de Altamira, efectuada por los métodos más modernos de la teconología química
}

Prof. Dr. ERICH PIETSCH RAFAEL MELIDA POCH, Dr. arquitecto FERNANDO AGUIRRE de YRAOLA, Dr. arquitecto

\section{simopsis}

Se describen en el presente artículo las fases de la instalación y el interesante proceso tecnológico seguido para la realización de la copia del techo de la gran sala de la cueva de Altamira, que el Gobierno de la República Federal Alemana ha regalado al pueblo de Madrid, y que puede visitarse en el jardín del Museo Arqueológico Nacional.

En este trabajo se pone una vez más de relieve la asombrosa calidad de los métodos científicos alemanes, que constituye un feliz ejemplo de la aportación de la técnica a la conservación de joyas artísticas, así como también de la estrecha colaboración realizada por los técnicos alemanes y españoles. 
Las maravillosas pinturas de la cueva de Altamira, en la provincia de Santander, fueron descubiertas por don Marcelino de Sautuola en 1879 y constituyen, como es sabido, la joya del arte rupestre paleolítico, a la par que uno de los ejemplos más antiguos del empleo por el hombre de los colores minerales (30.000-15.000 años a. d. J. C.).

Precisamente esta última razón fue la que determinó al profesor Dr. Erich Pietsch, eminente químico alemán, Director del Instituto Gmelin, de la Max Planck Gesellschaft, de Frankfurt, y al Director del Departamento de Química del Deutsches Museum, de Munich, a reproducir el techo de la gran sala de la cueva de Altamira, como punto central del material a exponer en la Sección dedicada a la Prehistoria de la Tecnología Química en la célebre Institución alemana.

En 1958, el Dr. Pietsch, en representación del Deutsches Museum, pidió permiso al Estado Español para realizar los trabajos de copia y aceptó las condiciones impuestas por las autoridades españolas: la de no tocar el techo en absoluto, ya que las pinturas originales presentan aún, tras muchos milenios, la misma humedad de filtraciones que el primer día, y la de ejecutar la copia por partida doble para que quedara una en España. La primera condición hizo muy difíciles los trabajos de reproducción. El techo es una superficie accidentada, una conformación de la que emergen prominencias con diferencias máximas de 34 centímetros.

Después de haber estudiado 19 cuevas en Francia y en España, el Dr. Pietsch y su esposa, la Dra. Gisela Fietsch, procedieron a tomar fotografías estereogramétricas desde unos 30 puntos, mediante un procedimiento parecido al de la fotografía aérea. De cada punto obtuvieron un par en blanco y negro, y otra en color. De los negativos en color, tras un minucioso examen de confrontación con escalas cromáticas, se sacaron copias y ampliaciones.

La evaluación de los pares estereoscópicos se verificó en el Instituto de Geodesia Aplicada, de Frankfurt, sobre estereoplanígrafos, obteniéndose planos de curvas de nivel. Mediante

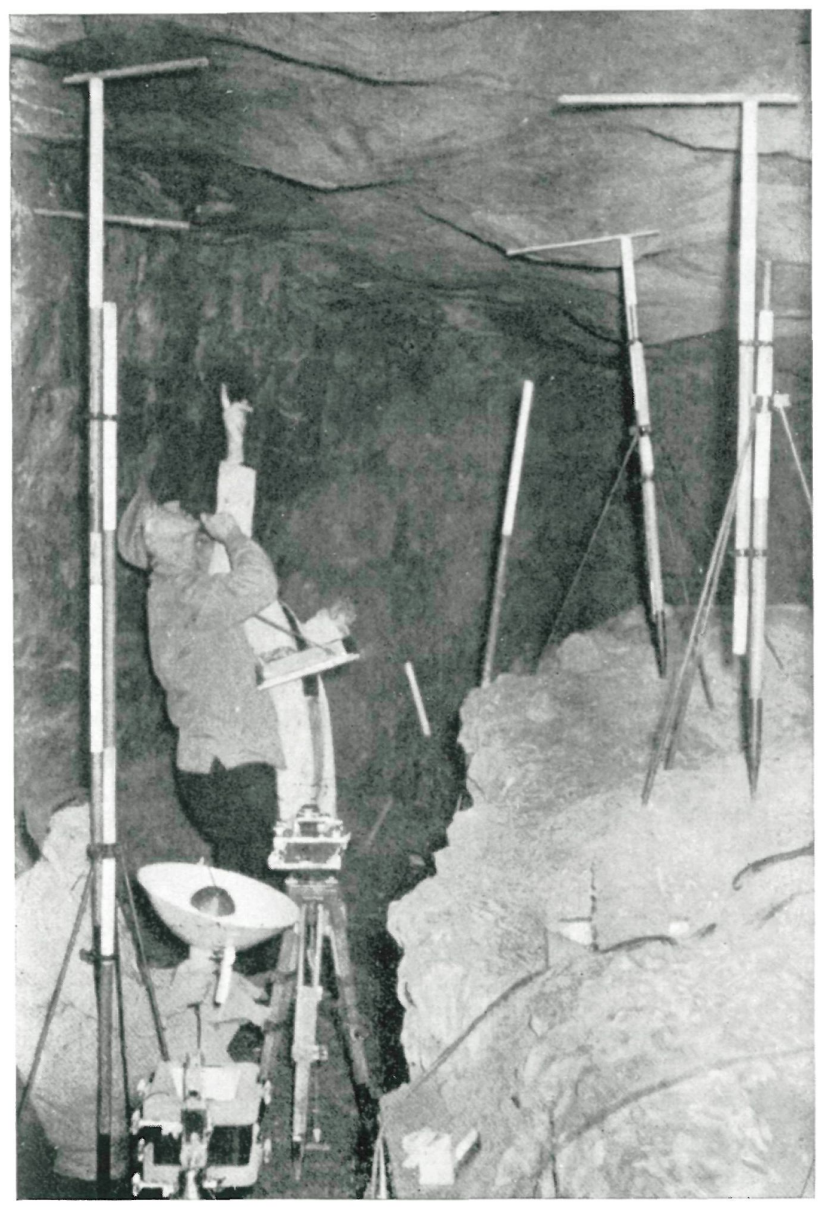
la aplicación de medidas adecuadas, se pudo conseguir la integración de cada par en un plano de referencia $y$, por lo tanto, una imagen total del techo en blanco y negro. Después, utilizando hojas de curvas de nivel, se realizaron fragmentariamente bloques de relieve negativo en bruto, en yeso, por medio de una máquina grabadora de precisión, según el principio del pantógrafo. Acoplando estas piezas de cuatro en cuatro, se obtuvo un vaciado que reproducía en bloque de $1 \times 1 \mathrm{~m}$ del techo de Altamira. Como no era posible la ejecución de los mínimos detalles estructurales del techo sobre la superficie de un material tan quebradizo como el yeso, se cubrió el vaciado con una capa de plastilina de $3 \mathrm{~mm}$ de espesor, con lo que ya se tenía una superficie apta para las posteriores fases del trabajo.

Sobre los bloques se proyectó la correspondiente diapositiva en color, lanzada desde un proyector montado sobre un banco óptico, de modo que cada fragmento de la imagen proyectada incidiera sobre la correspondiente zona del bloque en bruto, lo que permitió reproducir sobre la plastilina los elementos de la estructura a partir de la imagen. Una vez realizados los cincuenta bloques, fueron fotografiados, obteniéndose ampliaciones a la mitad de tamaño del bloque original, las cuales fueron confrontadas con el techo original durante una expedición de control a Altamira. 

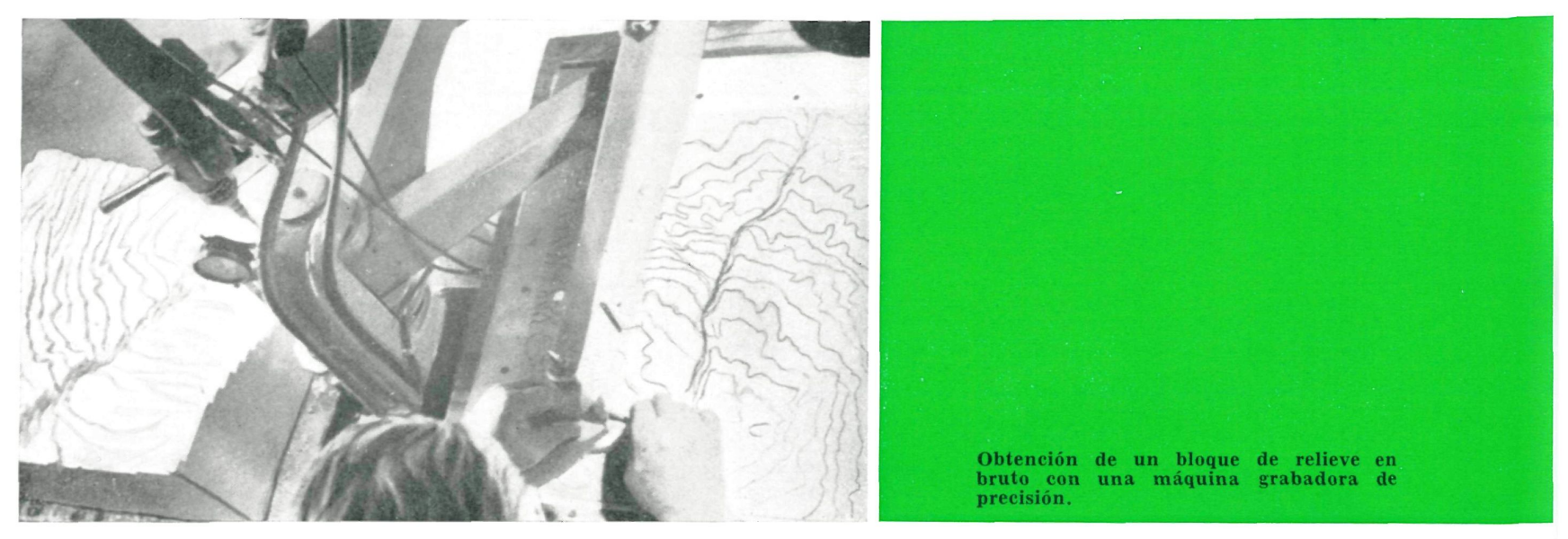

El siguiente paso de este formidable trabajo técnico fue conseguir un molde para obtener la copia final.

Después de variados y laboriosos trabajos de experimentación, se eligió una película de caucho silicón, trasdosada con un lecho de yeso estabilizador. La lechada, diluida en una proporción de volumen $3: 1$ con tricloroetileno (1,3 l tricloroetileno para $6 \mathrm{~kg}$ de caucho) y mezclada con la materia endurecedora TL (6 por 100 de yeso), fue cubriendo los $44 \mathrm{~m}^{2}$ de techo en diversas fases y capas. Sobre la capa total se montó un armazón de madera de $2 \times 2,5 \mathrm{~m}$, que había de servir para alojar los lechos de yeso. En la fase definitiva fue enrollada al modo de una alfombra y transportada así desde la gran nave de los talleres del Deutsches Museum hasta la sala de expociones y depositada en los elementos de yeso de dimensiones adecuadas para su transporte, los cuales habían sido invertidos y acoplados, y que habían de servir más tarde, una vez obtenida la primera copia en Munich, para realizar una segunda en Madrid.

En 1962, el Director General de Bellas Artes, Prof. Nieto, y el entonces Comisario del Tesoro Artístico, Prof. Dr. Iñiguez, encargaron a los arquitectos, don Rafael Mélida y don Fernando Aguirre, el proyecto de una sala, en el Museo Arqueológico de Madrid, destinada a albergar la copia que el Gobierno de la República Federal Alemana ha regalado a Madrid con motivo del cuarto Centenario de su capitalidad.

Correspondiendo a la idea de crear en el visitante un clima misterioso en relación al sentido mágico del arte que iba a admirar, se pensó en la construcción de un recinto subterráneo situado en el jardín del museo.

Por lo tanto, se proyectó una escalinata que diera acceso a la sala subterránea a través de un pasadizo que, en unión de la entrada, estuviese formado por muros a base de grandes bloques irregulares de pieđra y de un techo, de forma también irregular, que recordase a las construcciones más antiguas.

En contraste con esta idea rectora de la concepción del acceso, la cámara que debía albergar la copia del techo de Altamira fue tratada de un modo totalmente abstracto. A este fin, la más escueta decoración e iluminación, dentro de un moderno sentido arquitectónico, dirigen y concentran la atención del visitante hacia las maravillosas pinturas.

Sin embargo, la realización de esta instalación no ha podido llevarse a cabo sin serias dificultades técnicas.

En diciembre de 1963, el Dr. Arquitecto Aguirre de Yraola, perteneciente al I. E. T. c. c., y profesor de Restauración de Monumentos e Historia de la Arquitectura, de la Escuela Superior de Arquitectura, se trasladó a Munich para estudiar la instalación de la primera copia en el Deutsches Museum.

A su regreso, en unión del Dr. Arquitecto Mélida Poch, profesor de Historia del Arte en la citada Escuela Superior, procedieron a la construcción del recinto subterráneo. La condición de respetar los árboles del jardín del Museo obligó a construir unos pórticos de hormigón armado y un sistema de pantallas curvas de ladrillos para contener el empuje de las tierras, además de realizar una cuidadosa impermeabilización, ya que se exigió también la reposición del césped, precisamente encima del techo. 


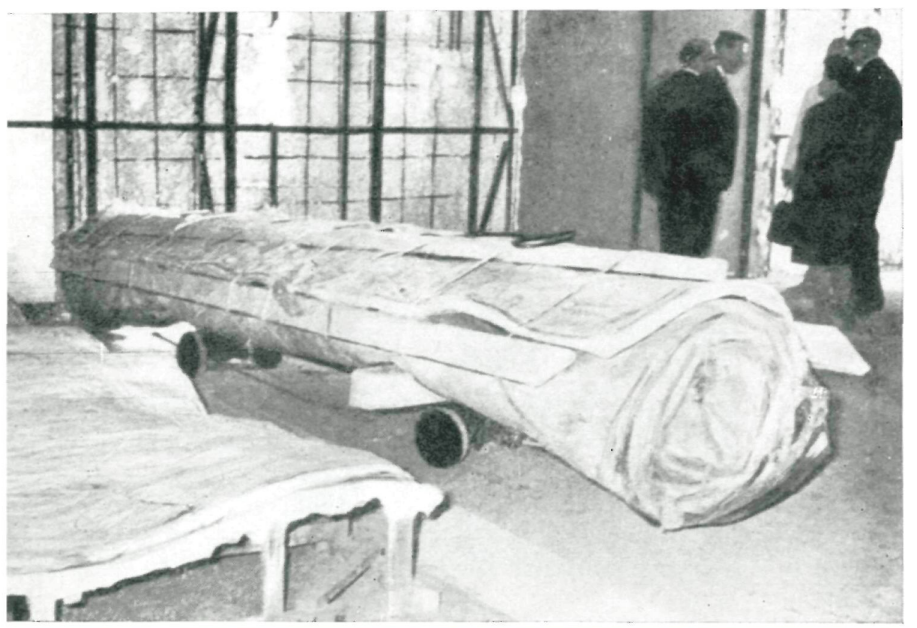

Capa de caucho silicón, dispuesta para ser trasladada.

Una vez comprobadas las condiciones estáticas y la resistencia del recinto, el equipo alemán, bajo la dirección del Dr. Pietsch, y formado por el ingeniero Sommerer, el escultor Widmer, el pintor Voglsamer y ayudantes, procedió a repetir la realización del vaciado definitivo. Se construyó una estructura, y sobre puntales de hierro fundido graduable se dispuso un soporte de chapas, sobre las que se acoplaron los lechos de yeso traídos de Alemania, y a los que ya se había adaptado el revestimiento de caucho silicón. Para la ejecución de la copia se utilizó, lo mismo que en Munich, la masa mineral sintética Dyckerhoff, compuesta de material calcáreo o marga caliza y de cemento portland blanco, y obtenida después de ha-

Pasadizo de entrada a la sala de exposición en el Museo Arqueológico Nacional.

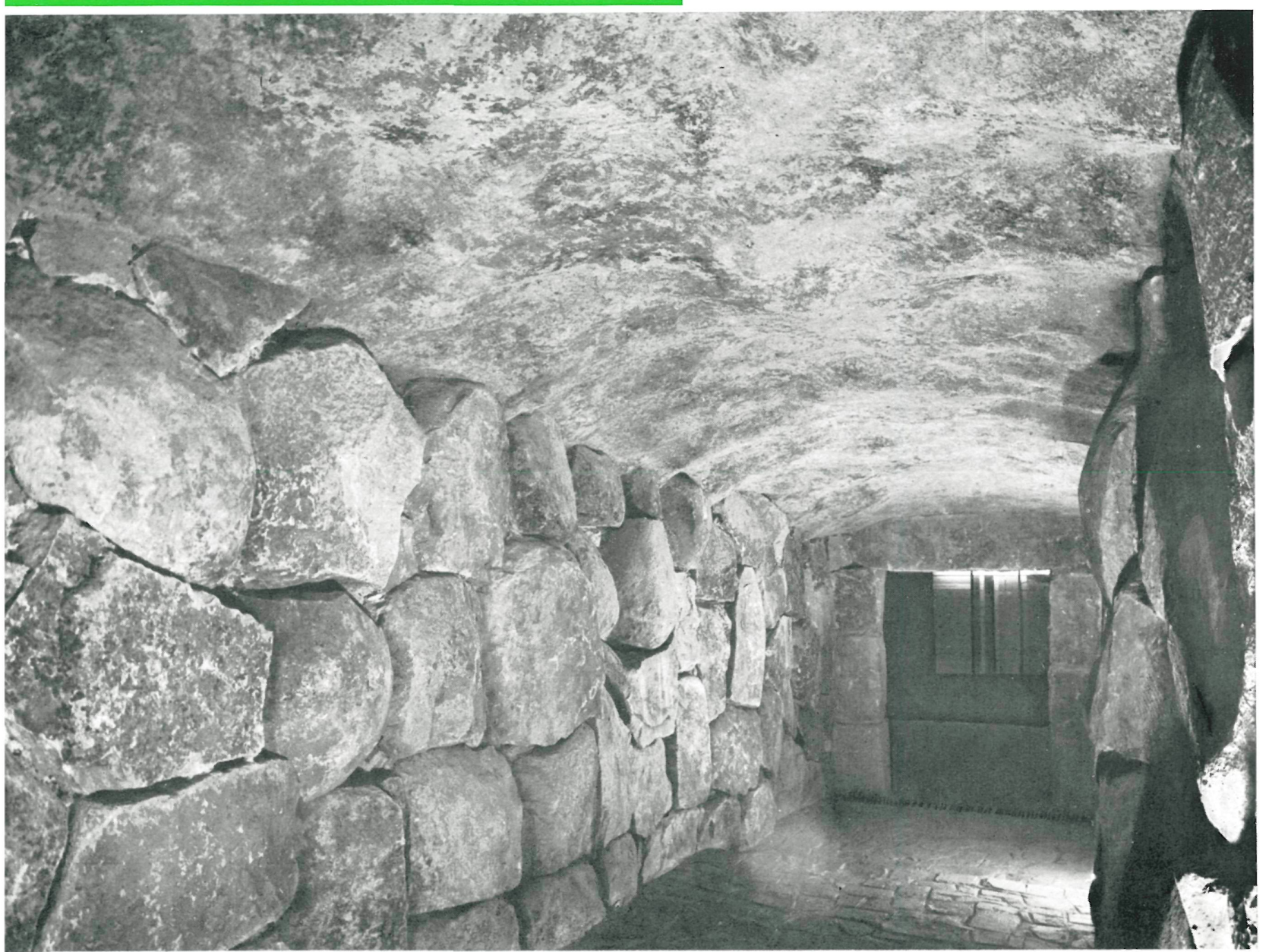




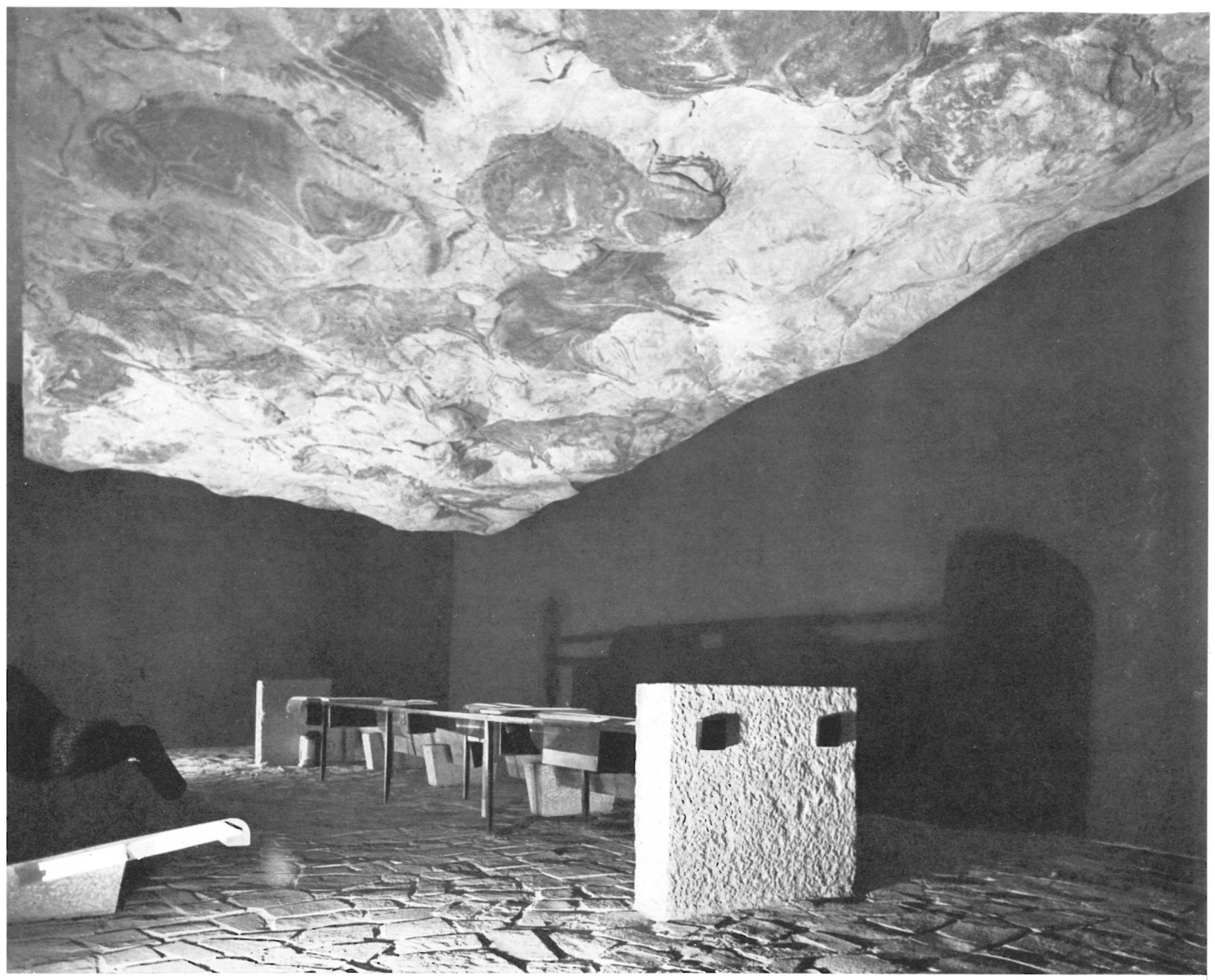

La reproducción en el recinto del jardín del Museo Arqueológico de Madrid, en vísperas de su inauguración.

ber analizado la composición química de un pequeño bloque extraído del recinto de Altamira. La copia reproduce, pues, sintéticamente, el material rocoso de la cueva.

La suspensión del vaciado final se llevó a cabo mediante un verdadero bosque de alambre de hierro galvanizado $(6 \mathrm{~km})$, sujetos a doscientos pares de garfios. El peso del techo es de 7 toneladas, aproximadamente.

La pintura de la copia se ejecutó según el procedimiento pictórico del Arte Paleolítico. Utilizando colores minerales en bruto se hicieron ensayos de color en numerosas partes de la pintura del techo, superponiéndose sobre estas pruebas reproducciones en color, y comprobando con el original de Altamira.

Por último, en el recinto madrileño se ha dispuesto una instalación de acondicionamiento de aire que permita mantener la valiosa copia en las óptimas condiciones higrotérmicas.

Los elevados gastos de este alarde de la tecnología moderna han corrido a cargo del Gobierno alemán y de diversas firmas comerciales alemanas. El Ministerio de Educación Nacional español, a través de la Dirección General de Bellas Artes, ha financiado la construcción de la nueva sala, cuyas dimensiones son aproximadamente $14 \times 5 \times 4 \mathrm{~m}$, sin contar el pasadizo, y que ha sido inaugurada solemnemente el 14 de octubre. 


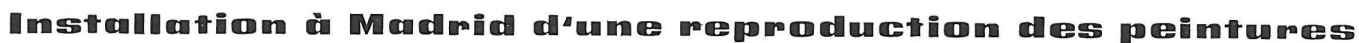

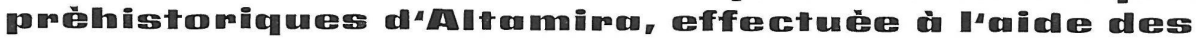 mókthodes les plus modemies de lat techmologie chimique}

Prof. Dr. Erich Pietsch; Rafael Mélida Poch, Dr. Architecte, et Fernando Aguirre de Yraola, Dr. Architecte.

Cet article décrit les phases d'installation et l'intéressant processus technologique suivi pour la réalisation de la copie du plafond de la grande salle de la grotte d'Altamira, offerte par le Gouvernement de la République fédérale d'Allemagne au peuple de Madrid, que l'on peut visiter dans le jardin du Musée Archéologique National de cette ville.

Dans cet article est mise, une fois de plus, en relief l'étonnante qualité des méthodes scientifiques allemandes, qui constituent un heureux exemple de l'apport de la technique à la conservation des trésors artistiques, ainsi que l'étroite collaboration réalisée par les techniciens allemands et espagnols.

\section{A meproduction in Mandrid of the Autramiren prehistromice mumol paintimgs, dome by the most modern procedures of chemical techmology}

Prof. Dr. Erich Pietsch; Dr. Archs. Rafael Mélida Poch and Fernando Aguirre de Yraola.

The article describes the various stages of preparing and carrying out the reproduction of the famous ceiling of the Altamira caves. The Government of the German Federal Republic has presented to the people of Madrid this reproduction, which can be admired in the garden of the National Archaeological Museum.

This feat is one more example of the very high quality of German technology. It is also a most happy contribution to the preservation of art treasures, and it has served as a further opportunity of cooperation between German and Spanish technicians and artists.

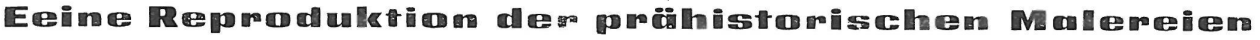

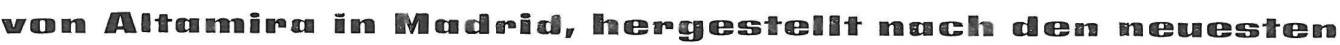

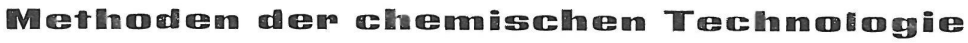

Prof. Dr. Erich Pietsch; Dr. Rafael Mélida Poch, Architekt, und Dr. Fernando Aguirre de Yraola, Architekt.

Im vorliegenden Artikel werden die einzelnen Phasen und der interessante technologische Prozess zur Her. stellung einer Kopie der Decke der Gran Sala aus der Höhle von Altamira beschrieben. Die Kopie ist ein Geschenk der Deutschen Bundesrepublik an das Spanische Volk und kann im Garten des Archäologischen Nationalmuseums in Madrid besichtigt werden.

Diese Arbeit zeigt wieder einmal die erstaunliche Qualität der deutschen wissenschaftlichen IMethoden und b'1det ein gelurgenes Beispiel für den Beitrag der Technik zur Erhaltung von Kunstschätzen und eine enge Zusammenarbeit zwischen deutschen und spanischen Technikern. 